\title{
SYNTHESIS AND EVALUATION OF NEW ARYLAZOLES DERIVATIVES AGAINST METHICILLIN RESISTANT BACTERIA
}

\author{
*Mohamed Hussein Hannoun, Mohamed Samy M. Hagras, Hamada S. Abulkhair, \\ Abdelrahman S. Mayhoub \\ Department of Pharmaceutical Organic Chemistry, Faculty of Pharmacy, Al-Azhar \\ University, Cairo, Egypt \\ *Corresponding author: Mohamehannoun.p.z@azhar.edu.eg
}

\begin{abstract}
:
In the previously reported arylthiazole antibiotics the lipophilic part of the nbutylphenyl moiety was replaced with naphthyl ring which improved its activity against Vancomycin resistant strains of Staphylococcus aureus. In the other hand, the incorporation of the $C=N$ linker connecting the nitrogenous head with thiazole within an oxadiazole ring provided orally available analogs with relatively long half-life. New eight derivatives of 2-(thiazol-5-yl)-1,3,4-oxadiazole was synthesized by combining both structural modifications in one new scaffold to enhance the pharmacokinetic profile and antibacterial activities against malicious microbes, two of them is comparably potent as Vancomycin.
\end{abstract}

keywords: Arylazoles, Antimicrobial, Oxadiazole, phenylthiazole

\section{INTRODUCTION}

\subsection{Overview:}

Bacterial resistance became one of the global crises.(Datta \& Huang, 2008) Causes that exacerbate this threat are disregarding pharmaceutical companies research in antimicrobial field, improper use of antibiotics in unnecessary cases and excessive use of antibiotics in agriculture.(Landers, Cohen, Wittum, \& Larson, 2012; Shapiro, Hicks, Pavia, \& Hersh, 2013) One of these bacteria is Staphylococcus aureus which developed resistance to penicillin-like antibiotics, Methicillin-Resistance $S$. aureus (MRSA) as methicillin antibiotic was widely used to eradicate it before its withdrawal from the market due to its toxicity.(Lowy, 2003) In addition to resistance to methicillin, resistance to other antibiotics like Vancomycin, Linezolid and Daptomycin, the refuge of MRSA treatment, were also appeared.(Husain, Rawat, Umesh, \& Verma, 2018) Due to that, a lot of efforts were done in developing new anti-MRSA and vaccination, however there are a necessity of developing new scaffold with a deferent cellular target.

\subsection{Phenylthiazoles as a Novel Class of Antimicrobial agents:}

Since the first invention of 2-phenylthiazoles as a new class of antibacterial in 2014, their advantage over the presence antibiotics were documented.(Mohammad et al., 2014; Mohammad, Reddy, et al., 2015) For instance, resistant mutant could not be isolated as a result of targeting two novel proteins in peptidoglycan pathway simultaneously.(Mohammad, Mayhoub, Cushman, \& Seleem, 2015; Nikolaidis, FaviniStabile, \& Dessen, 2014; Typas, Banzhaf, Gross, \& Vollmer, 2012) Essentially, their antibacterial potency covers many highly resistant strains (e.g. Vancomycin-resistant 
Staphylococcus aureus, Vancomycin-resistant enterococci, and many others).(Eid et al., 2017; Mohammad, Reddy, et al., 2015) So far, several modifications on the structure of the scaffold the lead compound $\mathbf{1}$ that furnished rigorous structure-activity and structure-kinetic relationships (SAR and SKR).(Mayhoub et al., 2012) Briefly, the moderate antibacterial activity of the lead compound $\mathbf{1}$ and its poor pharmacokinetic properties, being rapidly metabolized with very short half-life value, have been improved following two series of structures modifications; one at the lipophilic part (i.e. $n$-butylphenyl) and another set at the nitrogenous head (Figure 1).(Seleem et al., 2016) In this vein, replacement the lipophilic $n$-butylphenyl moiety with naphthyl ring improve the antibacterial effect especially on Vancomycin-resistant strains (Eid et al., 2017; Hagras, Abutaleb, et al., 2018; Mohammad et al., 2017; Seleem et al., 2016) (Figure 1). On the other hand, incorporating the $\mathrm{C}=\mathrm{N}$ linker, which connects the nitrogenous head of thiazole, within an oxadiazole ring provided orally available analogs with a biological half-life of almost 4-8 h.(Hagras, Hegazy, et al., 2018; Kotb et al., 2019) In addition, 1,3,4-oxadiazole is a pharmacophoric ring system, presented in several bactericidal compounds that are active against $S$. aureus strains including MRSA and Vancomycin-resistant S. aureus (VRSA).(Hagras, Abutaleb, et al., 2018; Hagras, Hegazy, et al., 2018; Spink et al., 2015; Z. Zheng et al., 2018) The synthesis of 1,3,4-oxadiazoles from carbohydrazide derivatives were reported by two different approaches. The first one includes the reaction with carboxylic acids in the presence of phosphorus trichloride (Taha, Imran, Rahim, Wadood, \& Khan, 2018; Taha, Ismail, Imran, Rokei, et al., 2015; Taha, Ismail, Imran, Selvaraj, et al., 2015; Taha et al., 2017), and the second approach involved the reaction between the acid hydrazide with carbon disulfide in basic media.(Q.-Z. Zheng et al., 2010) In this work, we combined both structural elements in one new scaffold to control both antibacterial activities vs. malicious microbes and enhance the overall pharmacokinetic profile. Eight derivatives, with the same structural elements, were synthesized and evaluated against MRSA.

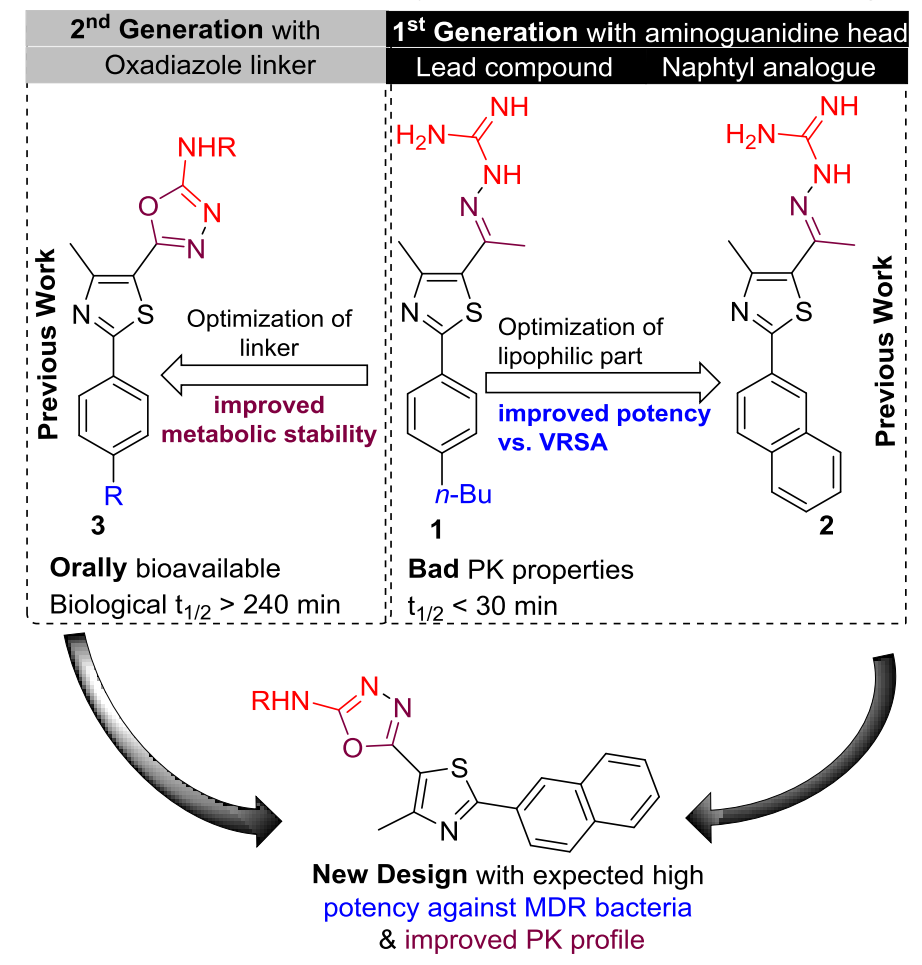

Figure 1. Design of naphthylthiazoles with oxadiazole linkers. 


\section{RESULTS AND DISCUSSION}

\subsection{Chemistry:}

Starting 2-(4-methyl-2-(naphthalen-2-yl)thiazol-5-yl)-5-(methylsulfonyl)-1,3,4 oxadiazole (9) was synthesized as outlined in Schemes 1. Naphthalene-2carbothioamide in absolute ethanol $(15 \mathrm{~mL})$ allowed to react with ethyl 2-chloro-3oxobutanoate under reflux for 4 hours. The resulting thiazole-5-carboxylate ethyl ester was treated with hydrazine hydrate in ethanol for 8 hours to obtain the carbohydrazide 6. Subsequent reflux of the latter with carbon disulfide in alcoholic solution of potassium hydroxide followed by stirring of the produced potassium salt with dimethyl sulfate for 2 hours afforded 2-(4-methyl-2-(naphthalen-2- yl)thiazol-5-yl)-5(methylthio)-1,3,4-oxadiazole (8). The methylsulfonyl derivative 9 was obtained through cautious addition of meta-chloroperoxybenzoic acid ( $m$-CPBA) onto a solution of 8 while stirring in DCM. Heating 9 with the appropriate amine afforded our new 2(thiazol-5-yl)- 1,3,4-oxadiazole derivatives (10-17).(Hannoun, Hagras, Kotb, El-Attar, \& Abulkhair, 2019)

\section{Scheme 1: Synthetic protocol of starting 2-(4-methyl-2-(naphthalen-}

\section{2-yl)thiazol-5-yl)-5-(methylsulfonyl)-1,3,4-oxadiazole}<smiles>[Z]c1nnc(-c2sc(-c3ccc4ccccc4c3)nc2C)o1</smiles>

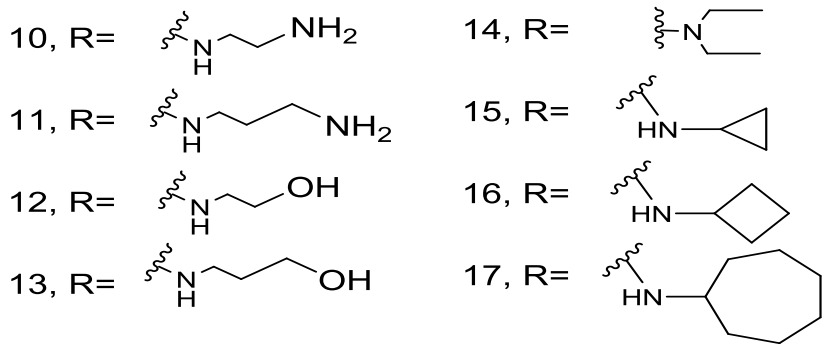

Reagents and conditions: (a) Absolute $\mathrm{EtOH}$, heat at reflux, $4 \mathrm{~h}$, (b) Absolute $\mathrm{EtOH}$, $\mathrm{NH}_{2} \mathrm{NH}_{2} \cdot \mathrm{H}_{2} \mathrm{O}$, heat at reflux, $8 \mathrm{~h}$; c) $\mathrm{CS}_{2}, \mathrm{KOH}, \mathrm{EtOH}$, heat at reflux, $12 \mathrm{~h}$; (d) dimethyl sulfate, $\mathrm{H}_{2} \mathrm{O}$, stirring at $23^{\circ} \mathrm{C}, 2 \mathrm{~h}$; (e) $m$-CPBA, dry DCM, $23^{\circ} \mathrm{C}, 16 \mathrm{~h}$; (f) appropriate amine, $\mathrm{K}_{2} \mathrm{CO}_{3}$, DMF, heat at $80{ }^{\circ} \mathrm{C}$ for $0.5-12 \mathrm{~h}$ 


\subsection{Experimental section:}

General. ${ }^{1} \mathrm{H}$ NMR spectra were run at $400 \mathrm{MHz}$ and ${ }^{13} \mathrm{C}$ spectra were determined at $100 \mathrm{MHz}$ in deuterated chloroform $\left(\mathrm{CDCl}_{3}\right)$, or dimethyl sulfoxide $\left(\mathrm{DMSO}-d_{6}\right)$ on a Varian Mercury VX-400 NMR spectrometer. Chemical shifts are given in parts per million (ppm) on the delta (d) scale. Chemical shifts were calibrated relative to those of the solvents. Flash chromatography was performed on 230-400 mesh silica. The progress of reactions was monitored with Merck silica gel IB2-F plates $(0.25$ $\mathrm{mm}$ thickness). The infrared spectra were recorded in potassium bromide disks on pye Unicam SP 3300 and Shimadzu FT IR 8101 PC infrared spectrophotometer. Mass spectra were recorded at $70 \mathrm{eV}$. Purity was accessed by High Performance Liquid Chromatography using acetonitrile-phosphate buffer 3:1. All yields reported refer to isolated yields.

\section{Ethyl 4-methyl-2-(naphthalen-2-yl)thiazole-5-carboxylate (5)}

Compound 4 (2.61 g, $14 \mathrm{mmol})$ and ethyl 2-chloro-3-oxobutanoate $(3.88 \mathrm{~mL}$, $4.62 \mathrm{~g}, 28 \mathrm{mmol})$ were added to absolute ethanol $(30 \mathrm{~mL})$. The reaction mixture was heated at reflux for $4 \mathrm{~h}$. After removal of solvent under reduced pressure, the solid residue was purified by crystallization from ethanol to provide the desired product as white crystals $(3.73 \mathrm{~g}, 90 \%) \mathrm{mp}=103{ }^{\circ} \mathrm{C} ;{ }^{1} \mathrm{H}$ NMR $\left(\mathrm{DMSO}-d_{6}\right) \delta: 8.62(\mathrm{~s}, 1 \mathrm{H}), 8.10$ $7.96(\mathrm{~m}, 4 \mathrm{H}), 7.59-7.56(\mathrm{~m}, 2 \mathrm{H}), 4.31(\mathrm{q}, J=7.6 \mathrm{~Hz}, 2 \mathrm{H}), 2.71(\mathrm{~s}, 3 \mathrm{H}), 1.30(\mathrm{t}, J=7.6$ $\mathrm{Hz}, 3 \mathrm{H}$ ); ${ }^{13} \mathrm{C}$ NMR (DMSO- $\left.d_{6}\right) \delta: 171.2,169.4,161.8,160.8,134.5,133.1,129.9$, 129.4, 129.3, 128.29, 128.23, 127.0, 123.8, 51.6, 20.9, 17.7; MS (m/z) 297.

\section{4-Methyl-2-(naphthalen-2-yl)thiazole-5-carbohydrazide (6)}

To a solution of 5 (1.19 g, $4 \mathrm{mmol})$ in ethanol $(15 \mathrm{~mL})$, hydrazine hydrate $(99 \%$, $1 \mathrm{~mL}, 20 \mathrm{mmol}$ ) was added dropwise. The reaction mixture was heated at reflux for $8 \mathrm{~h}$ then allowed to cool down to room temperature. The formed solid was separated by filtration and crystallized from ethanol to provide the desired product as yellow crystals $(1.62 \mathrm{~g}, 95 \%) \mathrm{mp}=195{ }^{\circ} \mathrm{C} ;{ }^{1} \mathrm{H}$ NMR (DMSO- $\left.d_{6}\right) \delta: 9.58$ (brs, $\left.1 \mathrm{H}\right), 8.52(\mathrm{~s}, 1 \mathrm{H}), 8.10$ $7.95(\mathrm{~m}, 4 \mathrm{H}), 7.59-7.56(\mathrm{~m}, 2 \mathrm{H}), 4.61(\mathrm{brs}, 2 \mathrm{H}), 2.62(\mathrm{~s}, 3 \mathrm{H}) ;{ }^{13} \mathrm{C}$ NMR (DMSO-d 6$) \delta$ : 171.2 , 166.3, 161.6, 155.3, 134.3, 133.2, 130.2, 129.4, 129.2, 128.2, 128.0, 127.5, 126.2, 123.8, 17.4; MS (m/z) 283.

\section{2-(4-Methyl-2-(naphthalen-2-yl)thiazol-5-yl)-5-(methylthio)-1,3,4-oxadiazole (8)}

Potassium hydroxide $(0.4 \mathrm{~g}, 10 \mathrm{mmol})$ was added to a solution of $\mathbf{6}(2.83 \mathrm{~g}, 10$ $\mathrm{mmol})$ in ethanol $(15 \mathrm{~mL})$, followed by drop-wise addition of carbon disulphide $(3 \mathrm{~mL}$, $110 \mathrm{mmol}$ ) over $0.5 \mathrm{~h}$. The reaction mixture was stirred at room temperature for an additional $15 \mathrm{~min}$ and then heated to reflux until the evolution of hydrogen sulfide gas ceased. After completion of the reaction, as monitored by TLC, the obtained intermediate 7 was poured on cold water $(50 \mathrm{~mL})$, filtered, washed with water, dried and crystallized from ethanol to provide yellow crystals $(3.15 \mathrm{~g}, 87 \%) \mathrm{mp}=248{ }^{\circ} \mathrm{C} ;{ }^{1} \mathrm{H}$ NMR (DMSO- $\left.d_{6}\right) \delta: 8.58(\mathrm{~s}, 1 \mathrm{H}), 8.08-7.94(\mathrm{~m}, 4 \mathrm{H}), 7.59-7.56(\mathrm{~m}, 2 \mathrm{H}), 2.67(\mathrm{~s}, 3 \mathrm{H})$; ${ }^{13} \mathrm{C}$ NMR (DMSO- $d_{6}$ ) $\delta: 177.1,168.6,156.9,156.3,134.5,133.1,129.6,129.5,129.3$, 128.3, 128.2, 127,6, 126.9, 123.8, 113.9, 17.6; MS (m/z) 362. The obtained yellow solid $7(0.76 \mathrm{~g}, 2.1 \mathrm{mmol})$ was dissolved in water $(15 \mathrm{~mL})$. Then, dimethyl sulfate $(0.5 \mathrm{~mL}, 4$ mmol) was added dropwise with vigorous stirring. After $2 \mathrm{~h}$, the formed solid was filtered and washed with copious amounts of water to yield the titled compound as a yellowish white solid $(0.64 \mathrm{~g}, 90 \%) ; \mathrm{mp}=155{ }^{\circ} \mathrm{C} ;{ }^{1} \mathrm{H}$ NMR $\left(\mathrm{DMSO}-d_{6}\right) \delta: 8.64(\mathrm{~s}, 1 \mathrm{H})$, 8.11-7.93 (m, 4H), 7.61- $7.58(\mathrm{~m}, 2 \mathrm{H}), 2.93(\mathrm{~s}, 3 \mathrm{H}), 2.73$ (s, 3H); MS (m/z) 339. 


\section{2-(4-Methyl-2-(naphthalen-2-yl)thiazol-5-yl)-5-(methylsulfonyl)-1,3,4-oxadiazole}

(9)

To a solution of $8(0.44 \mathrm{~g}, 1.3 \mathrm{mmol})$ in dry DCM $(5 \mathrm{~mL}), m$-CPBA $(0.514 \mathrm{~g}$, $2.9 \mathrm{mmol})$ diluted with DCM $(5 \mathrm{~mL})$ was added portion-wise with continuous stirring. Afterward, the reaction mixture was kept at $23{ }^{\circ} \mathrm{C}$ for $16 \mathrm{~h}$, additional DCM (10 mL) was added and the reaction mixture was washed with $25 \mathrm{~mL}$ of $5 \%$ aqueous solution of sodium metabisulfite, and $25 \mathrm{~mL}$ of $5 \%$ aqueous sodium carbonate. The organic layer was separated, dried and concentrated under reduced pressure to give the desired product as yellow crystals $(0.43 \mathrm{~g}, 90 \%) \mathrm{mp}=142{ }^{\circ} \mathrm{C} ;{ }^{1} \mathrm{H}$ NMR $\left(\right.$ DMSO- $\left.d_{6}\right) \delta: 8.63(\mathrm{~s}$, $1 \mathrm{H}), 8.06-7.86(\mathrm{~m}, 4 \mathrm{H}), 7.58-7.56(\mathrm{~m}, 2 \mathrm{H}), 3.70(\mathrm{~s}, 3 \mathrm{H}), 2.75(\mathrm{~s}, 3 \mathrm{H}) ;{ }^{13} \mathrm{C} \mathrm{NMR}$ (DMSO-d $)_{6} \delta$ : 169.6, 167.0, 161.6, 158.3, 134.6, 133.1, 129.5, 129.3, 128.9, 128.3, 128.2, 127.6, 127.1, 123.8, 113.7, 42.6, 17.8; MS (m/z) 371 .

\section{Compounds 10-17. General procedure.}

To a solution of $9(0.09 \mathrm{~g}, 0.25 \mathrm{mmol})$ in dry DMF $(5 \mathrm{~mL})$, appropriate amine was added. The reaction mixture was heated at $80{ }^{\circ} \mathrm{C}$ for $0.5-12 \mathrm{~h}$, and then poured over ice water $(50 \mathrm{~mL})$. The formed solid was extracted with ethyl acetate $(10 \mathrm{~mL})$. The organic layer was evaporated under reduced pressure. The obtained crude material was then purified by crystallization or column chromatography. Physical properties and spectral analysis of isolated products are listed below:

\section{$N^{1}$-\{5-[4-Methyl-2-(naphthalen-2-yl)thiazol-5-yl]-1,3,4-oxadiazol-2-yl\}ethane-1,2- diamine (10)}

Following the general procedure, and using ethylenediamine $(26.7 \mu \mathrm{L}, 0.4$ mmol), compound 10 was obtained as brown solid $(0.071 \mathrm{~g}, 81 \%) \mathrm{mp}=190{ }^{\circ} \mathrm{C} ;{ }^{1} \mathrm{H}$ NMR (DMSO- $\left.d_{6}\right) \delta: 8.54(\mathrm{~s}, 1 \mathrm{H}), 8.07-7.93(\mathrm{~m}, 4 \mathrm{H}), 7.77$ (brs, $\left.1 \mathrm{H}\right), 7.59-7.56(\mathrm{~m}, 2 \mathrm{H})$, 3.50-3.48 (m, 2H), $2.67(\mathrm{~s}, 3 \mathrm{H}), 2.59-2.55(\mathrm{~m}, 2 \mathrm{H}), 1.78$ (brs, $2 \mathrm{H}) ;{ }^{13} \mathrm{C}$ NMR (DMSO$\left.d_{6}\right) \delta: 166.4,164.2,153.9,153.0,134.3,133.2,129.9,129.4,129.1,128.2,128.0,127.5$, 126.4, 123.7, 115.5, 52.4, 37.5, 17.3; MS $(\mathrm{m} / \mathrm{z}) 351$.

$N^{1}$-\{5-[4-Methyl-2-(naphthalen-2-yl)thiazol-5-yl]-1,3,4-oxadiazol-2-yl\}propane-1,3diamine (11)

Following the general procedure, and using propane-1,3-diamine $(33.6 \mu \mathrm{L}, 0.4$ mmol), compound 11 was obtained as yellow solid $(0.066 \mathrm{~g}, 72 \%) \mathrm{mp}=175{ }^{\circ} \mathrm{C} ;{ }^{1} \mathrm{H}$ NMR (DMSO- $\left.d_{6}\right) \delta: 8.54(\mathrm{~s}, 1 \mathrm{H}), 8.07-7.93(\mathrm{~m}, 4 \mathrm{H}), 7.71(\mathrm{brs}, 1 \mathrm{H}), 7.59-7.56(\mathrm{~m}, 2 \mathrm{H})$, 3.45-3.42 (m, 2H), 2.68 (s, 3H), 2.58-2.56 (m, 2H), 1.67 (brs, $2 \mathrm{H}), 1.25-1.21(\mathrm{~m}, 2 \mathrm{H})$; ${ }^{13} \mathrm{C}$ NMR (DMSO- $d_{6}$ ) $\delta$ : 166.4, 164.2, 153.9, 153.0, 134.3, 133.2, 129.9, 129.4, 129.1, 128.2, 128.0, 127.5, 126.4, 123.7, 115.5, 42.7, 37.5, 35.0, 17.3; MS (m/z) 365 .

2-\{[5-(4-Methyl-2-(naphthalen-2-yl)thiazol-5-yl)-1,3,4-oxadiazol-2-yl]amino\}ethan1-ol (12)

Following the general procedure, and using 2-aminoethan-1-ol $(24.2 \mu \mathrm{L}, 0.4$ mmol), compound 12 was obtained as yellow solid $(0.069 \mathrm{~g}, 78 \%) \mathrm{mp}=165{ }^{\circ} \mathrm{C} ;{ }^{1} \mathrm{H}$ NMR (DMSO- $\left.d_{6}\right) \delta: 8.53(\mathrm{~s}, 1 \mathrm{H}), 8.07-7.92(\mathrm{~m}, 4 \mathrm{H}), 7.81$ (brs, $\left.1 \mathrm{H}\right), 7.56-7.53(\mathrm{~m}, 2 \mathrm{H})$, 4.76 (brs, 1H), 3.55-3.54 (m, 2H), 3.40-3.38 (m, 2H), $2.65(\mathrm{~s}, 3 \mathrm{H}),{ }^{13} \mathrm{C}$ NMR (DMSO$\left.d_{6}\right) \delta: 166.4,163.5,161.1,153.8,152.8,134.3,133.2,129.9,129.4,129.1,128.2,127.5$, 126.4, 123.7, 115.6, 59.3, 45.8, 17.0; MS $(\mathrm{m} / \mathrm{z}) 352$.

\section{3-\{[5-(4-Methyl-2-(naphthalen-2-yl)thiazol-5-yl)-1,3,4-oxadiazol-2-}

yl]amino\}propan-1-ol (13)

Following the general procedure, and using 3-aminopropan-1-ol $(30.6 \mu \mathrm{L}, 0.4$ mmol), compound 13 was obtained as yellow solid $(0.074 \mathrm{~g}, 81 \%) \mathrm{mp}=175{ }^{\circ} \mathrm{C} ;{ }^{1} \mathrm{H}$ 
NMR (DMSO- $\left.d_{6}\right) \delta: 8.53(\mathrm{~s}, 1 \mathrm{H}), 8.07-7.92(\mathrm{~m}, 4 \mathrm{H}), 7.85$ (brs, $\left.1 \mathrm{H}\right), 7.56-7.53(\mathrm{~m}, 2 \mathrm{H})$, 4.48 (brs, 2H), 3.50-3.47 (m, 2H), 3.27-3.23 (m, 2H), 2.66 (s, 3H), 1.73-1.71 (m, 2H); ${ }^{13} \mathrm{C}$ NMR (DMSO- $\left.d_{6}\right) \delta: 166.4,163.5,161.1,153.8,152.8,134.3,133.2,129.9,129.4$, $129.1,128.2,127.5,126.4,123.7,115.6,58.5,41.9,35.1,17.0 ;$ MS $(m / z) 366$.

\section{$N, N$-Diethyl-5-(4-methyl-2-(naphthalen-2-yl)thiazol-5-yl)-1,3,4-oxadiazol-2-amine} (14)

Following the general procedure, and using diethylamine $(41.3 \mu \mathrm{L}, 0.4 \mathrm{mmol})$, compound 14 was obtained as brown solid $(0.077 \mathrm{~g}, 85 \%) \mathrm{mp}=161{ }^{\circ} \mathrm{C} ;{ }^{1} \mathrm{H}$ NMR $\left(\mathrm{DMSO}-d_{6}\right) \delta: 8.57(\mathrm{~s}, 1 \mathrm{H}), 8.07-7.89(\mathrm{~m}, 4 \mathrm{H}), 7.58-7.49(\mathrm{~m}, 2 \mathrm{H}), 3.19(\mathrm{~m}, 4 \mathrm{H}), 2.66(\mathrm{~s}$, $3 \mathrm{H}), 1.12(\mathrm{~m}, 6 \mathrm{H}) ;{ }^{13} \mathrm{C}$ NMR (DMSO- $\left.d_{6}\right) \delta: 166.4,163.5,153.9,152.5,134.3,133.2$, $130.0,129.4,129.2$, 128.2, 128.0, 127.6, 126.4, 123.8, 115.6, 34.9, 22.5, 17.4; $\mathrm{MS}(\mathrm{m} / \mathrm{z})$ 364.

N-Cyclopropyl-5-[4-Methyl-2-(naphthalen-2-yl)thiazol-5-yl]-1,3,4-oxadiazol-2amine (15)

Following the general procedure, and using cyclopropylamine $(27.7 \mu \mathrm{L}, 0.4$ mmol), compound 15 was obtained as yellow solid $(0.078 \mathrm{~g}, 90 \%) \mathrm{mp}=155{ }^{\circ} \mathrm{C} ;{ }^{1} \mathrm{H}$ NMR (DMSO- $\left.d_{6}\right) \delta: 8.56(\mathrm{~s}, 1 \mathrm{H}), 8.09-7.94(\mathrm{~m}, 4 \mathrm{H}), 7.89$ (brs, $\left.1 \mathrm{H}\right), 7.58-7.53(\mathrm{~m}, 2 \mathrm{H})$, $2.69(\mathrm{~m}, 1 \mathrm{H}), 2.66(\mathrm{~s}, 3 \mathrm{H}), 1.19(\mathrm{~m}, 4 \mathrm{H}) ;{ }^{13} \mathrm{C}$ NMR $\left(\mathrm{DMSO}-d_{6}\right) \delta: 166.4,163.7,153.9$, $152.8,134.3$, 133.2,130.0, 129.4, 129.2, 128.2, 128.0, 127.5, 126.4, 123.8, 115.6, 31.2, 19.8, 17.4; MS $(\mathrm{m} / \mathrm{z}) 348$.

$\mathrm{N}$-Cyclobutyl-5-[4-methyl-2-(naphthalen-2-yl)thiazol-5-yl]-1,3,4-oxadiazol-2-amine (16)

Following the general procedure, and using cyclobutylamine $(34.1 \mu \mathrm{L}, 0.4$ mmol), compound 16 was obtained as yellow solid $(0.076 \mathrm{~g}, 84 \%) \mathrm{mp}=145{ }^{\circ} \mathrm{C} ;{ }^{1} \mathrm{H}$ NMR (DMSO- $\left.d_{6}\right) \delta: 8.56(\mathrm{~s}, 1 \mathrm{H}), 8.09-7.94(\mathrm{~m}, 4 \mathrm{H}), 7.86(\mathrm{brs}, 1 \mathrm{H}), 7.58-7.53(\mathrm{~m}, 2 \mathrm{H})$, $2.75(\mathrm{~m}, 1 \mathrm{H}), 2.66(\mathrm{~s}, 3 \mathrm{H}), 1.99(\mathrm{~m}, 4 \mathrm{H}), 1.69(\mathrm{~m}, 2 \mathrm{H}) ;{ }^{13} \mathrm{C}$ NMR (DMSO-d $) \delta: 166.4$, $163.7,153.9,152.8,134.3,133.2,130.0,129.4,129.2,128.2,128.0,127.5,126.4,123.8$, 115.6, 33.2, 20.8, 17.4, 14.1; MS $(\mathrm{m} / \mathrm{z}) 362$.

$\mathrm{N}$-cycloheptyl-5-(4-methyl-2-(naphthalen-2-yl)thiazol-5-yl)-1,3,4-oxadiazol-2amine (17)

Following the general procedure, and using cycloheptylamine $(51 \mu \mathrm{L}, 0.4$ mmol), compound 17 was obtained as yellow solid $(0.08 \mathrm{~g}, 79 \%) \mathrm{mp}=185{ }^{\circ} \mathrm{C} ;{ }^{1} \mathrm{H}$ NMR (DMSO- $\left.d_{6}\right) \delta: 8.56(\mathrm{~s}, 1 \mathrm{H}), 8.09-7.94(\mathrm{~m}, 4 \mathrm{H}), 7.79$ (brs, $\left.1 \mathrm{H}\right), 7.58-7.53(\mathrm{~m}, 2 \mathrm{H})$, $3.40(\mathrm{~m}, 1 \mathrm{H}) 2.71(\mathrm{~s}, 3 \mathrm{H}) 1.90(\mathrm{~m}, 2 \mathrm{H}) 1.70(\mathrm{~m}, 2 \mathrm{H}) 1.30(\mathrm{~m}, 4 \mathrm{H}) 1.17(\mathrm{~m}, 4 \mathrm{H}) ;{ }^{13} \mathrm{C}$ NMR (DMSO- $\left.d_{6}\right) \delta: 166.4,162.9,153.8,152.7,134.3,133.2,130.0,129.4,129.2$, 128.2 , 128.0, 127.6, 126.4, 123.8, 115.6, 52.3, 32.6, 25.6, 24.7, 17.4; MS (m/z) 404.

\subsection{Biological evaluation}

2.3.1. Method: MRSA clinical isolates (2658 RCMB) were obtained through the Regional Center of Mycology \& Biotechnology, Cairo, Egypt. The MICs of the newly synthesized compounds, tested against isolates of $S$. aureus, were determined by using the broth microdilution method in accordance with the Clinical and Laboratory Standards Institute guidelines. Bacteria were cultured in cation-adjusted Mueller Hinton broth in a 96-well plate. Compounds, using triplicate samples, were added to the plate and serially diluted. Plates were incubated at $37^{\circ} \mathrm{C}$ for 20 hours prior to determining the MIC. Plates were visually inspected and the MIC was categorized as the concentration 
at which no visible growth of bacteria was observed. The average of triplicate MIC determinations is reported.

2.3.2. Anti-MRSA activity: In the present study, eight derivatives of oxadizolylnaphthylthiazoles carrying a nitrogenous side chain at oxadiazole position- 5 were prepared, their antimicrobial activity was assessed against MRSA and the data were summarized in Table 1 . In brief, compounds that carrying hydrophilic terminal showed activity, while that without it the activity was demolished. Compounds that carry hydrophilic terminal group $\mathbf{1 0}$ and $\mathbf{1 1}$, amine group, showed moderate activity 7.8 $\mu \mathrm{g} / \mathrm{mL}$ without no effect on activity by increasing the length between the two nitrogen groups by one carbon atom. In contrast, alcoholic group showed promising activity against MRSA 2.9 and $3.2 \mu \mathrm{g} / \mathrm{mL}$ for compounds 12 and 13 respectively. In the other hand, lipophilic groups either small or large abolish the activity 14-17.

(1)

\section{CONCLUSION}

Bacterial resistance is one of the most serious problems nowadays. Among a lot of species that develop resistance to new antimicrobials, Methicillin resistance staph 
aureus come in the front of the list. A lot of effort was done to face this threat and one of them is the development that done by our lab.

After first reporting of 2-phenylthiazoles as antimicrobial agent a lot of modification was conducted on it the lead compound 1a. Replacement of the lipophilic part n-butylphenyl moiety with naphthyl ring improved its activity, while incorporating the $\mathrm{C}=\mathrm{N}$ linker, which connects the nitrogenous head with thiazole, within an oxadiazole ring provided orally available analogs with a biological longer half-life. We combined these two modification in one molecule to preserve antimicrobial activity and pharmacokinetics profile.

In this work, eight derivatives were synthesized, characterized and evaluated against malicious microbes. Two of them showed promising activity with MIC 2.9 and $3.2 \mu \mathrm{g} / \mathrm{ml}$. The two compounds contains terminal strong hydrophilic group (-OH).

\section{ACKNOWLEDGEMENT}

The authors would like to express their appreciation to Prof. Dr. Ashraf H. Bayoumi for his valuable support and sincere guidance throughout this work.

\section{REFERENCES}

Datta, R., \& Huang, S. S. (2008). Risk of infection and death due to methicillinresistant Staphylococcus aureus in long-term carriers. Clinical Infectious Diseases, 47(2), 176-181.

Eid, I., Elsebaei, M. M., Mohammad, H., Hagras, M., Peters, C. E., Hegazy, Y. A., . . A Abulkhair, H. S. (2017). Arylthiazole antibiotics targeting intracellular methicillin-resistant Staphylococcus aureus (MRSA) that interfere with bacterial cell wall synthesis. European journal of medicinal chemistry, 139, 665-673.

Hagras, M., Abutaleb, N. S., Ali, A. O., Abdel-Aleem, J. A., Elsebaei, M. M., Seleem, M. N., \& Mayhoub, A. S. (2018). Naphthylthiazoles: targeting multidrug-resistant and intracellular Staphylococcus aureus with biofilm disruption activity. ACS infectious diseases, 4(12), 1679-1691.

Hagras, M., Hegazy, Y. A., Elkabbany, A. H., Mohammad, H., Ghiaty, A., Abdelghany, T. M., ... . Mayhoub, A. S. (2018). Biphenylthiazole antibiotics with an oxadiazole linker: An approach to improve physicochemical properties and oral bioavailability. European journal of medicinal chemistry, 143, 14481456.

Hannoun, M. H., Hagras, M., Kotb, A., El-Attar, A.-A. M., \& Abulkhair, H. S. (2019). Synthesis and antibacterial evaluation of a novel library of 2-(thiazol-5yl)-1, 3, 4-oxadiazole derivatives against methicillin-resistant Staphylococcus aureus (MRSA). Bioorganic chemistry, 103364.

Husain, A., Rawat, V., Umesh, M. K., \& Verma, P. K. (2018). Vancomycin, linezolid and daptomycin susceptibility pattern among clinical isolates of methicillinresistant Staphylococcus aureus (MRSA) from Sub-Himalyan Center. Journal of laboratory physicians, 10(2), 145. 
Kotb, A., Abutaleb, N. S., Hagras, M., Bayoumi, A., Moustafa, M. M., Ghiaty, A., . .. Mayhoub, A. S. (2019). tert-Butylphenylthiazoles with an oxadiazole linker: a novel orally bioavailable class of antibiotics exhibiting antibiofilm activity. RSC advances, 9(12), 6770-6778.

Landers, T. F., Cohen, B., Wittum, T. E., \& Larson, E. L. (2012). A review of antibiotic use in food animals: perspective, policy, and potential. Public health reports, $127(1), 4-22$.

Lowy, F. D. (2003). Antimicrobial resistance: the example of Staphylococcus aureus. The Journal of clinical investigation, 111(9), 1265-1273.

Mayhoub, A. S., Marler, L., Kondratyuk, T. P., Park, E.-J., Pezzuto, J. M., \& Cushman, M. (2012). Optimization of thiazole analogues of resveratrol for induction of NAD $(\mathrm{P}) \mathrm{H}$ : quinone reductase $1(\mathrm{QR} 1)$. Bioorganic \& medicinal chemistry, 20(24), 7030-7039.

Mohammad, H., Mayhoub, A. S., Cushman, M., \& Seleem, M. N. (2015). Antibiofilm activity and synergism of novel thiazole compounds with glycopeptide antibiotics against multidrug-resistant Staphylococci. The Journal of antibiotics, 68(4), 259.

Mohammad, H., Mayhoub, A. S., Ghafoor, A., Soofi, M., Alajlouni, R. A., Cushman, M., \& Seleem, M. N. (2014). Discovery and characterization of potent thiazoles versus methicillin-and vancomycin-resistant Staphylococcus aureus. J Med Chem, 57(4), 1609-1615.

Mohammad, H., Reddy, P. N., Monteleone, D., Mayhoub, A. S., Cushman, M., \& Seleem, M. N. (2015). Synthesis and antibacterial evaluation of a novel series of synthetic phenylthiazole compounds against methicillin-resistant Staphylococcus aureus (MRSA). European journal of medicinal chemistry, 94, 306-316.

Mohammad, H., Younis, W., Chen, L., Peters, C. E., Pogliano, J., Pogliano, K., . . . Oldfield, E. (2017). Phenylthiazole antibacterial agents targeting cell wall synthesis exhibit potent activity in vitro and in vivo against vancomycinresistant Enterococci. J Med Chem, 60(6), 2425-2438.

Nikolaidis, I., Favini-Stabile, S., \& Dessen, A. (2014). Resistance to antibiotics targeted to the bacterial cell wall. Protein science, 23(3), 243-259.

Seleem, M. A., Disouky, A. M., Mohammad, H., Abdelghany, T. M., Mancy, A. S., Bayoumi, S. A., . . . Mayhoub, A. S. (2016). Second-generation phenylthiazole antibiotics with enhanced pharmacokinetic properties. J Med Chem, 59(10), 4900-4912. 
Shapiro, D. J., Hicks, L. A., Pavia, A. T., \& Hersh, A. L. (2013). Antibiotic prescribing for adults in ambulatory care in the USA, 2007-09. Journal of Antimicrobial Chemotherapy, 69(1), 234-240.

Spink, E., Ding, D., Peng, Z., Boudreau, M. A., Leemans, E., Lastochkin, E., . . . Testero, S. A. (2015). Structure-activity relationship for the oxadiazole class of antibiotics. J Med Chem, 58(3), 1380-1389.

Taha, M., Imran, S., Rahim, F., Wadood, A., \& Khan, K. M. (2018). Oxindole based oxadiazole hybrid analogs: Novel $\alpha$-glucosidase inhibitors. Bioorganic chemistry, 76, 273-280.

Taha, M., Ismail, N. H., Imran, S., Rokei, M. Q. B., Saad, S. M., \& Khan, K. M. (2015). Synthesis of new oxadiazole derivatives as $\alpha$-glucosidase inhibitors. Bioorganic \& medicinal chemistry, 23(15), 4155-4162.

Taha, M., Ismail, N. H., Imran, S., Selvaraj, M., Rahim, A., Ali, M., . . K Khan, K. M. (2015). Synthesis of novel benzohydrazone-oxadiazole hybrids as $\beta$ glucuronidase inhibitors and molecular modeling studies. Bioorganic \& medicinal chemistry, 23(23), 7394-7404.

Taha, M., Rahim, F., Imran, S., Ismail, N. H., Ullah, H., Selvaraj, M., . . Khan, K. M. (2017). Synthesis, $\alpha$-glucosidase inhibitory activity and in silico study of trisindole hybrid scaffold with oxadiazole ring: as potential leads for the management of Type-II diabetes mellitus. Bioorganic chemistry, 74, 30-40.

Typas, A., Banzhaf, M., Gross, C. A., \& Vollmer, W. (2012). From the regulation of peptidoglycan synthesis to bacterial growth and morphology. Nature Reviews Microbiology, 10(2), 123.

Zheng, Q.-Z., Zhang, X.-M., Xu, Y., Cheng, K., Jiao, Q.-C., \& Zhu, H.-L. (2010). Synthesis, biological evaluation, and molecular docking studies of 2chloropyridine derivatives possessing 1, 3, 4-oxadiazole moiety as potential antitumor agents. Bioorganic \& medicinal chemistry, 18(22), 7836-7841.

Zheng, Z., Liu, Q., Kim, W., Tharmalingam, N., Fuchs, B. B., \& Mylonakis, E. (2018). Antimicrobial activity of 1, 3, 4-oxadiazole derivatives against planktonic cells and biofilm of Staphylococcus aureus. Future medicinal chemistry, 10(3), 283-296. 


\section{"تشييد وتقييم مشتقات جديدة من الأريل أزول ضد البكتيريا المقاومة للميثيسيلين"}

*محد حسين محمد مهدي هنون ، محمد سامي محمد هجرس ، حماده السيد أبو الخير، عبد الرحمن صلاح ميهوب قسم الكيمياء العضوية ، كلية الصيدلة، جامعة الازهر ، القاهرة، مصر

Mohamehannoun.p.z@azhar.edu.eg : البريد الاليكتروني للباحث الرئيسي*

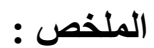

المقاومة البكتيرية هي واحدة من أخطر المشاكل في الوقت الحاضر. من بين الكثير من الأنواع التي تطور مقاومة

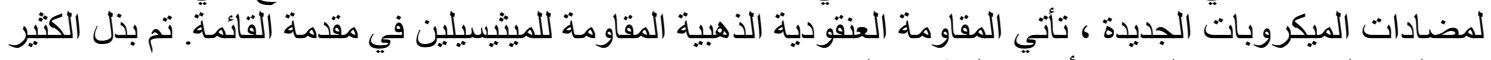

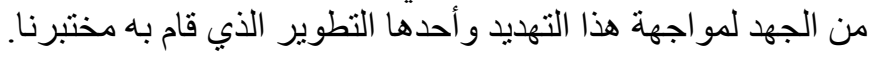

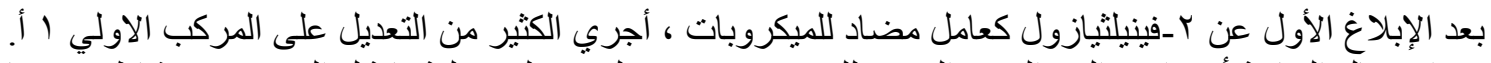

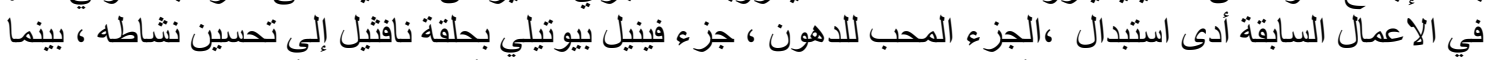

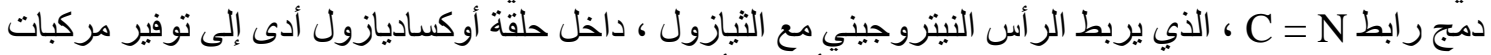

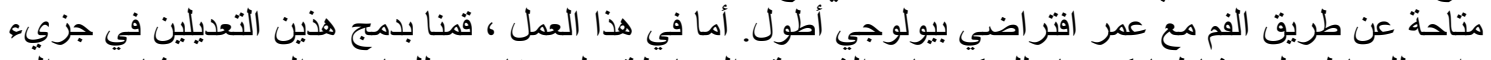

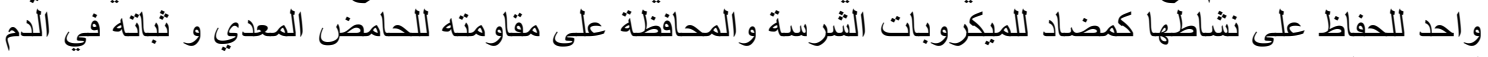
لفترة طويلة.

في هذا العمل ، تم تصنيع ثماي من المشتقات وتميز ها وتقييمها ضد الميكروبات الضارة. أظهر إثنان منهم نشاطًا وأعداً ضد البكتيريا المقاومة للميثيسيللين(2658RCMB).

الكلمات المفتاحية : أريلازول ، مضاد للميكروبات ، أوكساديازول ، فينيل ثيازول 